OPEN ACCESS

Edited by:

Xinyi Leng,

The Chinese University of Hong

Kong, China

Reviewed by:

Linfang Lan,

The First Affiliated Hospital of Sun

Yat-sen University, China

Yannan Yu,

University of California, Los Angeles,

United States

*Correspondence:

Shouchun Wang

wangsc13@163.com

Yi Yang

doctoryangyi@163.com

yang_yi@jlu.edu.cn

tThese authors have contributed equally to this work and share first authorship

Specialty section:

This article was submitted to Endovascular and Interventional Neurology,

a section of the journal

Frontiers in Neurology

Received: 23 September 2020 Accepted: 28 December 2020

Published: 16 February 2021

Citation:

Wang Z, Wang C, Li C, Shi M, Wang $S$ and Yang $Y$ (2021) Stenting for Symptomatic Intracranial Vertebrobasilar Artery Stenosis in Northeast of China: A Single-Center Study. Front. Neurol. 11:609286. doi: 10.3389/fneur.2020.609286

\section{Stenting for Symptomatic Intracranial Vertebrobasilar Artery Stenosis in Northeast of China: A Single-Center Study}

\author{
Zhongxiu Wang ${ }^{1,2,3 t}$, Chao Wang ${ }^{1,2,3 \dagger}$, Chao Li $^{1,2,3}$, Mingchao Shi ${ }^{1,2,3}$, Shouchun Wang ${ }^{1,2,3 *}$ \\ and Yi Yang ${ }^{1,2,3 *}$ \\ ${ }^{1}$ Department of Neurology, Stroke Center \& Clinical Trial and Research Center for Stroke, The First Hospital of Jilin University, \\ Changchun, China, ${ }^{2}$ China National Comprehensive Stroke Center, Changchun, China, ${ }^{3}$ Jilin Provincial Key Laboratory of \\ Cerebrovascular Disease, Changchun, China
}

Objective: We described the incidence of surgery-related complications to evaluate the safety of endovascular therapy for severe symptomatic intracranial vertebral basilar artery stenosis (IVBS) in our stroke center in Northeast of China.

Methods: Consecutive patients with symptomatic IVBS caused by $70-99 \%$ stenosis despite standard medical treatment of antiplatelet agents plus statin were enrolled. Either balloon-mounted stent or balloon predilation plus self-expanding stent was performed. Clinical adverse events such as stroke, transient ischemic attack (TIA), and death after the surgery were documented. Radiological events such as in-stent thrombosis, dissection, and guide-wire perforation during the process were recorded as complications as well. The baseline characteristics and outcomes of patients among different Mori types were compared.

Results: From January 2017 to December 2018, 97 patients with stroke or TIA due to intracranial IVBS were treated by stenting, including 30 patients with basilar artery (BA) stenosis, 55 patients with intracranial vertebral artery (V4) stenosis, and 12 patients with V4-BA stenosis. The primary events include two intracranial hemorrhage $(2.1 \%$, $2 / 97)$, seven ischemic events $(7.2 \%, 7 / 97)$, and two death $(2.1 \%, 2 / 97)$. The successful stent deployment rate was 98.9\% (96/97). The Apollo stents were used more for Mori A lesions. Self-expanding stents were more used in Mori $\mathrm{C}$ lesions. Mori $\mathrm{C}$ lesions were more vulnerable to endovascular procedure and showed higher rate of complications than $A(p=0.008)$ and $B$ type $(p=0.047)$.

Conclusion: A high technical success rate of IVBS stenting could be achieved, and the safety was acceptable, whereas Mori $\mathrm{C}$ lesions were more vulnerable to endovascular procedure and showed a higher rate of complications than A and B types.

\footnotetext{
Keywords: symptomatic intracranial vertebrobasilar artery stenosis, stenting, outcome, complication, safety
} 


\section{INTRODUCTION}

Posterior circulation stroke takes $\sim 25 \%$ of all ischemic stroke cases, and unlike anterior stroke, symptomatic vertebrobasilar atherosclerotic disease is more challenging and has a relatively higher annual recurrence of stroke despite standard medical treatment of antiplatelet agents and statin (1-3), and the symptoms caused by large artery stenosis or occlusion are often devastating. Also, it was associated with a risk of stroke $>20 \%$, as was shown in a pooled data analysis from two studies $(4,5)$. Stenting is becoming a promising therapeutic method for recurrent ischemic events refractory to best medical treatment, but there are also side effects such as procedure-related ischemic stroke or transient ischemic attack (TIA), intracranial hemorrhage, and even death $(6,7)$. Former researches such as the Stenting and Aggressive Medical Management for Preventing Recurrent Stroke (SAMMPRIS) study (8) and the Vitesse Intracranial Stent Study for Ischemic Therapy (VISSIT) trial (9) used stents, either self-expanding or balloon-expandable ones, and demonstrated higher perioperative stroke and death rate compared with medical therapy, showing less favorable outcome of this procedure. However, not only advanced devices have emerged, but also more experienced hands have grown, making this technique more applicable and favorable (10). Because of different anatomy and function, the posterior circulation arteries might have different characteristics compared with that of anterior circulation for endovascular treatment (1). Moreover, intracranial artery stenosis is more frequently seen in Asian population than in white and black patients (11), and the northeast area of China holds people with one of the highest rates of stroke in the world, whose profiles have rarely been published. Thus, we aimed to investigate the safety of stenting for patients with severe intracranial vertebral BA stenosis (IVBS) refractory to medical treatment.

\section{METHODS}

\section{Participants}

This study was approved by the ethics committee of the First Hospital of Jilin University. Written informed consent was obtained from all patients and/or their relatives. Patients' baseline characteristics and outcomes were collected. Inclusion criteria for endovascular stenting were as follows (5): (1) 18 to 85 years old; (2) vertebral artery V4 segment or basilar artery atherosclerotic stenosis $70 \%$ or greater as defined by the Warfarin Aspirin Symptomatic Intracranial Disease Trial criteria (12) and a lesion length of $\leq 15 \mathrm{~mm}$ on digital subtraction angiography (DSA), with normal distal vessel; (3) recent non-disabling stroke or TIA of the vertebrobasilar vascular territory within 90 days refractory to standard medical therapy (stroke or TIA recurrence due to severe stenosis of VA and basilar artery (BA) under strict control of risk factors such as hypertension and diabetes mellitus $[\mathrm{DM}]$ and more than 3 months' use of at least one antiplatelet drug and statin, or stroke or TIA recurrence due to hypoperfusion $<3$ months even under aggressive medical treatment: dual antiplatelet drugs and control of low-density lipoprotein cholesterol $<1.8 \mathrm{mmol} / \mathrm{L}$ or $>50 \%$ decrease); (4) a modified Rankin score (mRS) $\leq 3$; (5) at least one atherosclerotic risk factor (hypertension, DM, hyperlipidemia, and cigarette smoking); and (6) hypoplastic posterior communicating artery (PcomA) and/or posterior cerebral artery P1 segment, and for V4 segment lesion, the contralateral vertebral artery should be hypoplastic or occluded. Exclusion criteria included (1) non-atherosclerotic stenosis; (2) patients with stroke symptoms that were not thromboembolic or hemodynamic (including perforator strokes); (3) intracranial hemorrhage in the territory of the stenotic artery within 6 weeks; (4) potential source of cardiac embolism; (5) concurrent intracranial tumor, aneurysm, and cerebral arteriovenous malformation; (6) tandem $\geq 50 \%$ stenosis of extracranial carotid or vertebral artery; (7) known contraindication to heparin, aspirin, clopidogrel, anesthesia, and contrast media; (8) platelet count $<100,000$; (9) international normalized ratio $>1.5$ (irreversible) and uncorrectable bleeding diathesis; (10) and life expectancy $<1$ year because of other medical conditions.

We use Mori classification of intracranial artery stenosis to differentiate lesion morphology (13): type A ( $<5 \mathrm{~mm}$ in length, concentric or moderately eccentric lesions not totally occlusive), type B (tubular, $5-10 \mathrm{~mm}$ in length, extremely eccentric or totally occluded lesions), and type C (10-15 $\mathrm{mm}$ in length, extremely angulated lesions with excessive tortuosity of the proximal segment, or totally occluded lesions). The medical treatment for risk factor control was based on the SAMMPRIS study and the Chinese ischemic stroke guideline. Clinical and radiological adverse events such as stroke, TIA, death, in-stent thrombosis, dissection, and guide-wire perforation were recorded as complications. Technical success was defined angiographically as $<30 \%$ residual stenosis (5). TIA was defined as a reversible neurological deficit that lasts for at least $10 \mathrm{~min}$ and completely resolved within $24 \mathrm{~h}$ regardless of the diffusionweighted imaging changes.

\section{Outcome Measures}

The primary outcome was any stroke (including ischemic or hemorrhagic), TIA, and death caused by the endovascular procedure during hospital stay. The secondary outcome was successful revascularization (residual stenosis <30\%) rate, 90-day mRS of patients with complications after stenting. Other radiological events, including in-stent thrombosis and dissection, were also recorded as adverse events. If a new posterior circulation stroke was suspected, computed tomography $(\mathrm{CT})$ or magnetic resonance imaging scans were arranged for documentation.

\section{Follow-Up}

Radiological and clinical follow-up information of patients with complications after surgery was obtained during in hospital. Ninety-day clinical outcomes were reviewed and collected by a trained neurologist who was blinded to treatment via face-to-face or telephone interview.

\section{Statistical Analysis}

Statistics analysis was performed with the SPSS version 16.0 (SPSS, Chicago, IL, USA). The baseline, imaging, and stenting 
TABLE 1 | Complication profile and prognosis.

\begin{tabular}{|c|c|c|c|c|c|c|c|c|c|c|c|}
\hline No. & Gender & Age & $\begin{array}{l}\text { Meri } \\
\text { type }\end{array}$ & Target artery & Intervention method & Complication & Remedial measure & Pre-NIHSS & Post-NIHSS & $\begin{array}{l}\text { New symptoms after } \\
\text { stenting }\end{array}$ & $90 \mathrm{~d} \mathrm{mRS}$ \\
\hline 1 & M & 60 & $\mathrm{~B}$ & V4-BA & Ballon mounted stent & Perforator injury & Drug therapy & 0 & 0 & Numbness of left extremities & 0 \\
\hline 2 & M & 68 & $\mathrm{~B}$ & V4 & Ballon + balloon mounted stent & Perforator injury & Drug therapy & 2 & 2 & Nystagmus, nausea & 0 \\
\hline 3 & M & 47 & $\mathrm{~B}$ & V4 & Ballon mounted stent & Thrombosis & IA urokinase & 0 & 0 & None & 0 \\
\hline 4 & M & 52 & A & V4 & Ballon + self-expending stent & Hemorrhage & BP control & 0 & 0 & Mild headache & 0 \\
\hline 5 & M & 70 & B & V4 & Ballon + self-expending stent & Perforator injury & Drug therapy & 2 & 2 & Mild weakness of right limbs & 0 \\
\hline 6 & $\mathrm{~F}$ & 71 & B & V4 & Ballon + self-expending stent & Perforator injury & Drug therapy & 0 & 0 & $\begin{array}{l}\text { Stroke recurrence } 2 \text { months } \\
\text { later }\end{array}$ & 3 \\
\hline 7 & M & 54 & A & V4 & Ballon + self-expending stent & Perforator injury & Drug therapy & 0 & 35 & Coma & 6 \\
\hline 8 & $\mathrm{~F}$ & 61 & C & BA & Fail & Hemorrhage & Protamine + BP control & 2 & 25 & $\begin{array}{l}\text { Paralysis, disturbance of } \\
\text { consciousness, dyspnea }\end{array}$ & 6 \\
\hline 9 & M & 55 & B & V4 & Ballon + self-expending stent & Thrombosis & IA urokinase & 0 & 0 & Dizziness, nausea & 1 \\
\hline 10 & M & 53 & $A$ & V4 & Ballon + self-expending stent & Thrombosis & IA urokinase & 0 & 0 & Transient dysarthria & 0 \\
\hline 11 & M & 49 & B & BA & Ballon + self-expending stent & Perforator injury & Drug therapy & 2 & 15 & Right limbs paralysis & 5 \\
\hline 12 & M & 61 & C & BA & Ballon + self-expending stent & Dissection & Stenting & 1 & 9 & Right limbs paralysis & 5 \\
\hline 13 & M & 66 & A & V4 & Ballon + self-expending stent & Perforator injury & Drug therapy & 2 & 3 & Dysarthria, dizziness & 2 \\
\hline 14 & M & 44 & $\mathrm{~B}$ & V4 & Balloon mounted stent & Thrombosis & Mechanical thrombectomy & 0 & 3 & Ataxia, nystagmus & 2 \\
\hline 15 & M & 59 & A & BA & Ballon + self-expending stent & Dissection & Stenting & 0 & 0 & None & 0 \\
\hline 16 & $\mathrm{~F}$ & 54 & $\mathrm{~B}$ & $\mathrm{BA}$ & Ballon + self-expending stent & Hyper-perfusion & BP control & 1 & 1 & Headache, restlessness & 0 \\
\hline 17 & $\mathrm{~F}$ & 65 & $A$ & V4 & Ballon + self-expending stent & Hyper-perfusion & BP control & 2 & 2 & Headache, nausea & 1 \\
\hline 18 & M & 67 & $\mathrm{~B}$ & BA & Ballon + self-expending stent & Thrombosis & IA tirofiban & 0 & 5 & Left limb and facial paralysis & 1 \\
\hline 19 & M & 59 & A & V4 & Ballon + self-expending stent & Thrombosis & IA tirofiban + urokinase & 4 & 6 & Right limbs paralysis & 3 \\
\hline 20 & M & 43 & B & V4-BA & Ballon + self-expending stent & Dissection & Stenting & 1 & 1 & $\begin{array}{l}\text { Transient paralysis of left } \\
\text { limbs }\end{array}$ & 2 \\
\hline 21 & M & 49 & $A$ & V4 & Ballon + self-expending stent & Thrombosis & IA urokinase & 0 & 1 & $\begin{array}{l}\text { Transient nystagmus, } \\
\text { nausea, dysarthria }\end{array}$ & 1 \\
\hline 22 & M & 53 & A & V4 & Ballon + self-expending stent & Thrombosis & A tirofiban + urokinase & 3 & 3 & None & 0 \\
\hline
\end{tabular}


data of all patients are presented as means $( \pm$ SD) or median interquartile range for continuous variables and number for categorical data. Continuous variables were tested with the Student $t$-test, whereas categorical data were tested with $\chi^{2}$ test or Fisher exact test (when the expected cell frequency was $<5$ ). When continuous variables had skewed distributions, the Mann-Whitney $U$-test was used to identify the difference in the continuous variables. The significant $P$-value was set at $<0.05$.

\section{RESULTS}

From January 2017 to December 2018, 97 patients (67 males, aged $64.4 \pm 8.6$ ) with stroke or TIA due to IVBS were treated by stenting, including 30 patients with BA stenosis, 55 patients with V4 stenosis, and 12 patients with V4-BA stenosis. Vertebrobasilar artery stenosis and poor collaterals were all confirmed by DSA before stenting.

The successful stent deployment rate was 98.9\% (96/97). Taking all the other adverse events into account, the rate of overall complication was $22.7 \%$ (22/97). And the primary events include two cases of intracranial hemorrhage $(2.1 \%, 2 / 97)$ : one was caused by wire penetration of BA during the surgery and the other one was due to intimal damage confirmed by postoperative CT scan. Seven ischemic events happened within $24 \mathrm{~h}$ after the surgery including one TIA and six strokes $(7.2 \%, 7 / 97)$, all caused by perforator injury: two happened in V4 group and five happened in BA group. Two patients died $(2.1 \%$, 2/97) during hospital stay, one was caused by subarachnoid hemorrhage due to wire penetration, and the other one was caused by medulla oblongata infarction due to perforator injury. Other complications included eight $(8.2 \%, 8 / 97)$ patients with thrombosis during the procedure process, three $(3.1 \%, 3 / 97)$ patients with dissection after balloon dilation, and two $(2.1 \%$, 2/97) patients with hyperperfusion. Among all the 22 patients, nine $(40.9 \%)$ showed deterioration by increasing at least one National Institutes of Health Stroke Scale (NIHSS) score during in-hospital stay, whereas 17 patients $(77.3 \%)$ recovered to independence $(\mathrm{mRS}<3$ ) (Table 1$)$ in 90 days' follow-up.

The baseline characteristics of patients with or without surgery-related complications are presented in Table 2. We found no statistical differences on rate of hypertension, DM, coronary heart disease, atrial fibrillation, prior ischemic stroke, smoking, and alcohol drinking between these two groups.

As for whether the site of the stent would influence the rate of complication, we found no difference among patients who had stenting for BA $(6 / 30, p=0.508), \mathrm{V} 4(14 / 55, p=0.716)$, or V4-BA $(2 / 12, p=1)$. Nor did we find statistical difference of interventional techniques (balloon-mounted stent or balloon+ balloon-mounted stent or balloon+ self-expanding stent) in these segments of arteries on complication occurrence (3/23 vs. $1 / 6$ vs. $17 / 66 ; p=0.53$ vs. 1.0 vs. 0.086 ).

While Mori C lesions were more vulnerable to endovascular procedure and showed higher rate of complications than A type $(p=0.008)$ and B type $(p=0.047)$. Tables 3,4 show that as for $\mathrm{BA}$ and Mori $\mathrm{C}$ type lesion stenting, self-expanding stent such as Wingspan or Enterprise were more frequently applied ( $p=$
TABLE 2 | Clinical characteristics and complications.

\begin{tabular}{|c|c|c|c|c|}
\hline \multirow[t]{2}{*}{ Characteristic } & & \multicolumn{2}{|c|}{ Complication } & \multirow[t]{2}{*}{$P$} \\
\hline & & $\begin{array}{c}\text { Yes } \\
n=22\end{array}$ & $\begin{array}{c}\text { No } \\
n=75\end{array}$ & \\
\hline Age & $y \pm S D$ & $57.3 \pm 8.3$ & $58.9 \pm 8.5$ & 0.423 \\
\hline Male sex & $\%(n)$ & $72.7(16)$ & $68.0(51)$ & 0.673 \\
\hline Coronary heart disease & $\%(n)$ & $22.7(5)$ & $16.0(12)$ & 0.329 \\
\hline Atrial fibrillation & $\%(n)$ & 13.6 (3) & $10.7(8)$ & 0.708 \\
\hline Cerebral infarction/TIA & $\%(n)$ & $18.2(4)$ & $22.7(17)$ & 0.451 \\
\hline Hypertension & $\%(n)$ & $81.8(18)$ & $61.3(46)$ & 0.075 \\
\hline Diabetes mellitus & $\%(n)$ & $45.5(10)$ & $26.7(20)$ & 0.08 \\
\hline Alcohol drinking & $\%(n)$ & $50.0(11)$ & $59.2(45)$ & 0.404 \\
\hline Cigarette smoking & $\%(n)$ & $59.0(13)$ & $48.0(36)$ & 0.421 \\
\hline BA & $n$ & 6 & 24 & 0.508 \\
\hline V4 & $n$ & 14 & 41 & 0.716 \\
\hline V4-BA & $\mathrm{n}$ & 2 & 10 & 1 \\
\hline Balloon-mounted stent & $n$ & 3 & 20 & 0.53 \\
\hline Balloon + balloon-mounted stent & $\mathrm{n}$ & 1 & 5 & 1 \\
\hline Balloon + self-expanding stent & $\mathrm{n}$ & 17 & 49 & 0.086 \\
\hline Mori A & $\mathrm{n}$ & 5 & 33 & 0.538 \\
\hline Mori B & $n$ & 7 & 29 & 0.047 \\
\hline Mori C & $\mathrm{n}$ & 10 & 13 & $0.008^{\star}$ \\
\hline Lesion length & $\mathrm{mm} \pm \mathrm{SD}$ & $8.9 \pm 4.16$ & $6.8 \pm 3.4$ & 0.141 \\
\hline Stenosis percentage & $\% \pm \mathrm{SD}$ & $86.4 \pm 7.69$ & $84.7 \pm 8.21$ & 0.787 \\
\hline
\end{tabular}

${ }^{\star} P<0.05$ for comparing between two groups.

TABLE 3 | Lesion distribution and stenting method.

\begin{tabular}{lccc}
\hline Lesion location & Self-expanding stent & Balloon-mounted stent & $\boldsymbol{P}$-value \\
\hline BA & 25 & 4 & $0.029^{*}$ \\
V4 & 35 & 20 & 0.843 \\
V4-BA & 8 & 4 & 0.202
\end{tabular}

${ }^{\star} P<0.05$ for comparing between two groups.

TABLE 4 | Lesion type and stenting method.

\begin{tabular}{lccc}
\hline Lesion type & Self-expanding stent & Balloon-mounted stent & $\boldsymbol{P}$-value \\
\hline A & 20 & 18 & $0.006^{\star}$ \\
B & 29 & 6 & 0.980 \\
C & 19 & 4 & $0.018^{\star}$
\end{tabular}

${ }^{\star} P<0.05$ for comparing between two groups.

0.029, $p=0.018$ ). Compared to types $\mathrm{B}$ and $\mathrm{C}$ lesions, Mori $\mathrm{A}$ and V4 lesions were more likely to receive balloon-mounted stent $(p=0.006)$.

\section{DISCUSSION}

The rate of overall complications in our study was $22.7 \%$ $(22 / 97)$, which was higher than that reported in literature (14), because we included both radiological and clinical abnormalities 
during and after the procedure. Patients with radiological complications may not necessarily present clinical symptoms, but these abnormalities could compromise the integrity of the vessel wall or interfere the blood flow especially when occurring on the opening of an artery $(15,16)$. Thus, we took into account all these radiological changes as complications even if they did not necessarily show clinical symptoms as to present the characteristics of a lesion. For instance, we found type $\mathrm{C}$ lesions were more vulnerable to endovascular procedure and showed a higher rate of these complications, which means higher risks of symptom deterioration or new infarction that also have a clinical meaning: It is a reminder that we could not be too cautious when dealing with it.

Previous studies have shown that stenting for IVBS was challenging because of its higher complication rate and severe symptoms once it happened. Levy et al. (17) treated 11 IVBS patients; three suffered periprocedural deaths and one died of pontine stroke. Tsang et al. (18) conducted a systematic review and random-effects meta-analysis of all available randomized controlled trials evaluating the safety and efficacy of percutaneous transluminal angioplasty and stenting (PTAS), in comparison with medical therapy, for symptomatic intracranial artery stenosis (sICAS) and found a higher risk of any stroke or death within 2 years in the sICAS subgroup located in posterior circulation than medical treatment. SAMMPRIS (8) and VISSIT trial (9) both demonstrated higher perioperative stroke and death rate compared with medical therapy, showing less favorable outcome of this procedure. It was obvious that stenting for IVBS was no easy thing, and complications could occur; none of these results recommended stenting as first-line treatment for IVBS, but with the advancement of neurointervention devices and more experienced neurointerventionists, safety of PTAS for IVBS seemed to be acceptable. Miao et al. (19) treated 159 intracranial atherosclerotic disease (ICAD) patients with balloon-mounted stent and 141 ICAD patients with balloon plus self-expanding stent. The 30 -day rate of stroke, TIA, and death was $4.3 \%$. Ding et al. (15) analyzed 19 ICAD stenting series after SAMMPRIS trial, including 2,196 patients with 2,314 lesions, showing the median rate of postprocedural ischemic events was 9.4\% (range, 0-25\%). Thus, we believe that after a thorough clinical assessment and exquisite surgery procedure, a particular group of patients would benefit from IVBS stenting with high successful rate of stent deployment and low rate of complication occurrence such as stroke or death.

We collected and compared clinical data of patients with or without any kind of complications in order to distinguish patients who might suffer from surgery-related complications. As for medical history, we found no statistical difference of these factors between groups, but patients with hypertension and DM seemed to have a higher occurrence of complications, which explains that these patients have higher rates of perforator stroke and poor collateral status.

The practice that perforator stroke was more likely to happen after BA stenting was also reported in previous literatures (19). We assumed that compared with intracranial vertebral arteries, the BA had much more perforating branches and poor collateral circulation, which made it extremely sensitive to ischemia. Also, neurological deficits caused by the occlusion of perforator orifice of BA were severe and sometimes even lethal. This may be caused by atherosclerotic debris being displaced over the perforator origins during angioplasty or stent deployment. The SAMMPRIS trial concluded that the occlusion of perforating arteries was the most common cause of ischemic stroke especially after BA PTAS.

The rate of successful stent deployment in our center was 98.9\% (96/97), and device selection of self-expandable stent or balloon-mounted stent depended on arterial access and lesion morphology (7, 20). From our experience, the GatewayWingspan system with its excellent flexibility in traversing curvatures is more suitable for tortuous lesions, whereas the Apollo stent is preferred for patients with smooth arterial access, which does not require exchanging. Therefore, for patients with smooth arterial access and Mori A lesion, the balloonmounted stent would be convenient. For patients with tortuous arterial access and a Mori B or C lesion, Gateway balloon plus self-expandable stents such as Wingspan stent system is preferred. And if perforator arteries originated near the stenotic site, predilation of small-sized balloon plus self-expandable stent is also preferred out of protection for the orifice of the perforator artery, as we believed that balloon-mounted stent might hurt the perforator arteries more easily, which could cause devastating result. Following these easy strategies while doing the endovascular procedure, we did not find difference of complication rate among patients who had stenting for BA, V4, or V4-BA. Nor did we find any difference of interventional techniques (balloon-mounted stent or balloon + self-expanding stent) in these segments of artery. But we did have seven ischemic events happened, including one TIA and six strokes, all caused by perforator injury. Among all the 22 patients, nine (40.9\%) showed NIHSS deterioration by increasing at least 1 point. Two patients died $(2.1 \%, 2 / 97)$ : one was caused by subarachnoid hemorrhage due to wire penetration, and the other one was caused by medulla oblongata infarction due to perforator injury, whereas 17 patients $(77.3 \%)$ recovered to independence $(\mathrm{mRS}<3)$. Patients may show symptoms such as dizziness, nausea, and nystagmus, and mostly reached recession very soon. Our study showed even IVBS was challenging because of its higher complication rate; the majority could recovery to independency.

Some limitations should be considered when interpreting the findings because this is a single-center observational study with a relatively small size of patients. And we analyzed only the clinical data we collected during the hospital stay; thus, the long-term prognosis of this treating strategy such as stroke recurrence or restenosis is still to be investigated. Nevertheless, we described our experience and findings of stenting for IVBS in the population of northeast China.

\section{CONCLUSIONS}

A high technical success rate of IVBS stenting could be achieved, and the safety was acceptable, whereas Mori C lesions were more vulnerable to endovascular procedure and showed higher rate of complications than A and B types. 


\section{DATA AVAILABILITY STATEMENT}

The raw data supporting the conclusions of this article will be made available by the authors, without undue reservation.

\section{ETHICS STATEMENT}

The studies involving human participants were reviewed and approved by Human and Research Ethics committees of the First Hospital of Jilin University. The patients/participants provided their written informed consent to participate in this study.

\section{REFERENCES}

1. Zhang Q, Dong K, Song H. Comparison of stent versus medical therapy for symptomatic patients with intracranial atherosclerotic stenosis: a metaanalysis. J Neurol Sci. (2017) 372:272-278. doi: 10.1016/j.jns.2016.11.064

2. Xu S, Wu P, Shi H, Ji Z, Dai J. Hyperperfusion Syndrome after stenting for intracranial artery stenosis. Cell Biochem Biophys. (2015) 71:153742. doi: 10.1007/s12013-014-0377-7

3. Abuzinadah AR, Alanazy MH, Almekhlafi MA, Duan Y, Zhu H, Mazighi M, et al. Stroke recurrence rates among patients with symptomatic intracranial vertebrobasilar stenoses: systematic review and meta-analysis. J Neurointerv Surg. (2016) 8:112-6. doi: 10.1136/neurintsurg-2014-011458

4. Wang $\mathrm{T}$, Wang $\mathrm{X}$, Yang $\mathrm{K}$, Zhang J, Luo J. Endovascular treatment for symptomatic intracranial artery stenosis: protocol for a systematic review and network meta-analysis. BMJ Open. (2018) 8:e022359. doi: 10.1136/bmjopen-2018-022359

5. Mardighian D, Castellan L, Resta F, Piano M G, Comelli S, Barletta L, et al. Stenting for symptomatic intracranial vertebrobasilar artery stenosis: 30-day results in a high-volume stroke center. J Neurointerv Surg. (2016) 143:132-8. doi: 10.1016/j.clineuro.2016.02.029

6. Yu Jia Z, Sun Song Y, Jon Sheen J, Goo Kim J. Endovascular recanalization of symptomatic non-acute intracranial artery occlusion: procedural and midterm clinical outcomes in the anterior circulation. Interv Neuroradiol. (2019) 25:380-389. doi: 10.1177/1591019919826616

7. Bai WX, Gao BL, Li TX, Wang ZL, Cai DY, Zhu LF, et al. Wingspan stenting can effectively prevent long-term strokes for patients with severe symptomatic atherosclerotic basilar stenosis. Interv Neuroradiol. (2016) 22:318-24. doi: 10.1177/1591019915623797

8. Chimowitz MI, Lynn MJ, Derdeyn CP, Turan TN, Fiorella D, Lane BF, et al. Stenting versus aggressive medical therapy for intracranial arterial stenosis. $N$ Engl J Med. (2011) 365:993-1003. doi: 10.1056/NEJMoa1105335

9. Zaidat OO, Fitzsimmons BF, Woodward BK, Wang Z, Killer-Oberpfalzer M, Wakhloo A, et al. Effect of a balloon-expandable intracranial stent vs medical therapy on risk of stroke in patients with symptomatic intracranial stenosis: the VISSIT randomized clinical trial. Jama. (2015) 313:12408. doi: 10.1001/jama.2015.1693

10. Wang L, Shi W, Su Z, Liu X, Su H, Liu J, et al. Endovascular treatment of severe acute basilar artery occlusion. J Clin Neurosci. (2015) 22:1958. doi: 10.1016/j.jocn.2014.05.032

11. Wong LK. Global burden of intracranial atherosclerosis. Int J Stroke. (2006) 1:158-9. doi: 10.1111/j.1747-4949.2006.00045.x

12. Prognosis of patients with symptomatic vertebral or basilar artery stenosis. The Warfarin-Aspirin Symptomatic Intracranial Disease (WASID) study group. Stroke. (1998) 29:1389-92. doi: 10.1161/01.STR.29.7.1389

\section{AUTHOR CONTRIBUTIONS}

ZW and CW conceived and designed the study, acquired the data, and drafted and revised the manuscript. All authors analyzed and interpreted the data and critically revised the manuscript for important intellectual content.

\section{FUNDING}

This project was supported by the National Key R\&D Program of China (2016YFC1301600), JLUSTIRT (2017TD-12) and Jilin Provincial Key Laboratory (20190901005JC) to YY.

13. Mori T, Fukuoka M, Kazita K, Mori K. Follow-up study after intracranial percutaneous transluminal cerebral balloon angioplasty. AJNR Am J Neuroradiol. (1998) 19:1525-33.

14. Zhou Y, Wang L, Zhang JR, Zhou K, Shuai J, Gong ZL, et al. Angioplasty and stenting for severe symptomatic atherosclerotic stenosis of intracranial vertebrobasilar artery. Health Technol Assess. (2019) 63:1721. doi: 10.1016/j.jocn.2019.02.017

15. Ding D, Starke RM, Crowley RW, Liu KC. Role of stenting for intracranial atherosclerosis in the post-SAMMPRIS era. Biomed Res Int. (2013) 2013:304320. doi: 10.1155/2013/304320

16. Cheng L, Jiao L, Gao P, Song G, Chen S, Wang X, et al. Risk factors associated with in-hospital serious adverse events after stenting of severe symptomatic intracranial stenosis. Clin Neurol Neurosurg. (2016) 147:5963. doi: 10.1016/j.clineuro.2016.05.019

17. Levy EI, Horowitz MB, Koebbe CJ, Jungreis CC, Pride GL, Dutton K, et al. Transluminal stent-assisted angiplasty of the intracranial vertebrobasilar system for medically refractory, posterior circulation ischemia: early results. Neurosurgery. (2001) 48:1215-21. doi: 10.1227/00006123-20010600000002

18. Tsang FCP, Ho WS, Lee R, Leung GKK, Lui WM, Tsivgoulis $\mathrm{G}$, et al. Percutaneous transluminal angioplasty and stenting for symptomatic intracranial arterial stenosis: a systematic review and metaanalysis. Clin Neuroradiol. (2016) 9:351-8. doi: 10.1177/17562856166 50357

19. Miao Z, Zhang Y, Shuai J, Jiang C, Zhu Q, Chen K, et al. Thirtyday outcome of a multicenter registry study of stenting for symptomatic intracranial artery stenosis in China. Stroke. (2015) 46:2822-9. doi: 10.1161/STROKEAHA.115.010549

20. Park SC, Cho SH, Kim MK, Kim JE, Jang WY, Lee MK, et al. Long-term outcome of angioplasty using a wingspan stent, post-stent balloon dilation and aggressive restenosis management for intracranial arterial stenosis. Clin Neuroradiol. (2019) 30:159eur. doi: 10.1007/s00062-019-00793-1

Conflict of Interest: The authors declare that the research was conducted in the absence of any commercial or financial relationships that could be construed as a potential conflict of interest.

Copyright (C) 2021 Wang, Wang, Li, Shi, Wang and Yang. This is an open-access article distributed under the terms of the Creative Commons Attribution License (CC $B Y)$. The use, distribution or reproduction in other forums is permitted, provided the original author(s) and the copyright owner(s) are credited and that the original publication in this journal is cited, in accordance with accepted academic practice. No use, distribution or reproduction is permitted which does not comply with these terms. 